\title{
Advance on the preparation, physiological function and nanocarriers of resveratrol
}

\author{
Lingyi $\mathrm{Li}^{1}$ \\ ${ }^{1}$ College of Food Science, Southwest University, 400715 Chongqing, China; National Demonstration Center for Experimental Food \\ Science and Technology Education (Southwest University), 400715 Chongqing, China
}

\begin{abstract}
Resveratrol is a natural compound that has many beneficial physiological functions. In recent years, the research on resveratrol has gradually become a hotspot. This article summarized the literature reports all over the world, classified and concluded the extraction technology, function and nano-delivery carriers of resveratrol. Solvent extraction, microwave- and ultrasonic-assisted extraction, supercritical carbon dioxide extraction and enzymatic hydrolysis method were respectively discussed in detail. Then the antioxidant property, antibacterial activity, antiumor effect and the prevention of cardiovascular disease of resveratrol were also discussed. Finally, the resveratrol-loaded nanocarriers, such as nanoparticles, nanoemulsions, solid lipid particles and nanosuspensions were summarized. And the future research direction of resveratrol were prospected, in order to provide references and orientations for the further development and research of resveratrol.
\end{abstract}

\section{Introduction}

Resveratrol is a natural non-flavonoid polyphenol compound containing stilbene structure. It is a phytotoxin produced by plants in response to infection by the pathogen Botrytis cinerea ${ }^{[1]}$. It is also induced in response to a variety of stress conditions, such as vicissitudes in climate, exposure to ozone, sunlight and heavy metals ${ }^{[2]}$. It was first obtained from the root of Veratrum by Takaoka in $1940^{[3]}$, but since then it has not received enough attention. It was not until the emergence of the "French Paradox" phenomenon that it gradually attracted attention ${ }^{[4-5]}$. So far, it has been detected in more than 70 plant species, including grapes, peanuts, berries, and foods derived from them ${ }^{[6-8]}$. Fresh grape skin contains about 50 to $100 \mu \mathrm{g}$ of resveratrol per gram wet weight ${ }^{[9]}$, which contributes to a relatively high concentration of resveratrol in red wine and grape juice ${ }^{[8]}$.

Resveratrol, the chemical name is 3, 5, 4'trihydroxystilbene, with molecular formula $\mathrm{C}_{14} \mathrm{H}_{12} \mathrm{O}_{3}$, molecular weight is 228.25 , it is a colorless needle crystal, tasteless, hardly soluble in water, easily soluble in ether and trichloro methane, methanol, ethanol, acetone, ethyl acetate and other organic solvents. It have a melting point of $256 \sim 258^{\circ} \mathrm{C}$ and a sublimation temperature of $261^{\circ} \mathrm{C}$ [10-11]. It can show red color with alkaline solutions such as ammonia. It also can react chromogenic reaction with ferric chloride-potassium ferricyanide, this property can be used to identify resveratrol. Natural resveratrol has two structures, cisand trans- resveratrol. In nature, it mainly exists in trans conformation. The two structures can be combined with glucose to form cis- and trans-resveratrol glycosides, cisand trans-resveratrol glycosides can release resveratrol under the action of glycosidases in the intestine ${ }^{[12]}$. The molecular formula of resveratrol and its glycosides is shown in Figure 1. The cis-resveratrol has strong photosensitivity and is extremely unstable. The biological activity of its trans-isomer is stronger than that of the cis-isomer. Under ultraviolet light, the transresveratrol can be converted to the cis-isomer. In nature, plants mainly contain trans-resveratrol, cis-resveratrol glycosides and trans-resveratrol glycosides ${ }^{[13-15]}$. A large number of studies have found that resveratrol has a variety of pharmacological activities, such as anti-tumor, antioxidant, antibacterial, cardiovascular system protection, anti-inflammatory, etc. ${ }^{[16-23]}$, Therefore, resveratrol has great potential in the development of functional foods and drugs.

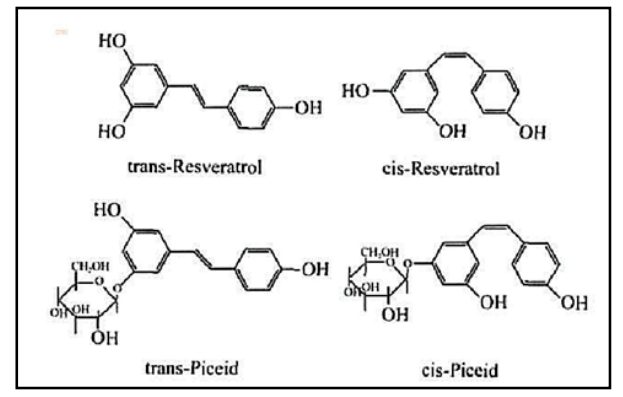

Fig. 1. Resveratrol and its glycoside molecular formula.

In recent years, because of its diverse pharmacological activities and characteristics, resveratrol has begun to be extracted in clinical trials and is widely used in various related fields. Therefore, it is

\footnotetext{
* Lingyi Li: 1006895596@qq.com
} 
an urgent need to develop the extraction method of resveratrol. Resveratrol has photosensitivity in solution, which is manifested as the conversion of transresveratrol to cis-resveratrol under sunlight or ultraviolet light irradiation. At the same time, it is also sensitive to $\mathrm{pH}$ and prone to oxidation reactions. The extracted resveratrol is usually unstable, easily oxidizable and sensitive to heat and light, which limits its application in the food industry ${ }^{[24-25]}$. In addition, its poor water solubility, fast metabolism, and low bioavailability make it difficult to exert good biological activity both in vitro and in vivo experiments ${ }^{[26]}$, which not only causes waste of resources and damages the environment, but also limits the popularization and application of resveratrol in the medicine and functional food ${ }^{[27-28]}$.

In recent years, the application of nanotechnology to improve the solubility of poorly soluble drugs and improve the bioavailability has received widespread attention ${ }^{[29-30]}$. Among them, the nano drug delivery system has great potential in improving the bioavailability of lipophilic drugs ${ }^{[31-33]}$. Liposomes, nanogels, polymer micelles, etc. were used as nanocarriers and combined with resveratrol to prepare nanomedicine. The schematic diagram of different nanocarriers used for resveratrol delivery was shown in Figure $2^{[34]}$. The embedding effect of nanocarriers on resveratrol achieves the purpose of slow release, prolongs its residence time in the gastrointestinal tract, improves its water solubility, physicochemical stability and metabolic stability, and improves its bioavailability.

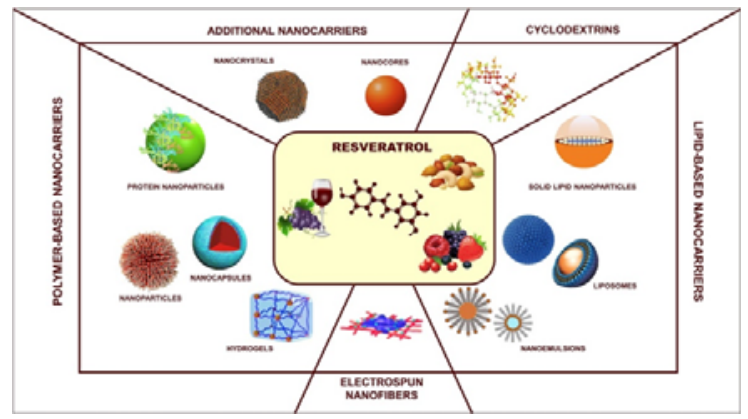

Fig. 2. Schematic diagram of different nanocarriers used for resveratrol delivery ${ }^{[34]}$.

In the paper, the preparation technology and functional activities of resveratrol, as well as the nanocarriers for encapsulating resveratrol were summarized and reviewed, which could provide reference and insight into the further research of resveratrol.

\section{Preparation technology of resveratrol}

At present, solvent extraction, microwave- and ultrasonic-assisted extraction, supercritical carbon dioxide extraction and enzymatic hydrolysis method were the main preparation technology of resveratrol.

\subsection{Solvent extraction method}

Solvent extraction is a method that uses the difference in solubility of substances to make the resveratrol separated from other components. Water, ethanol, methanol, ethyl acetate and acetone, etc. are commonly used as extraction solvents. In the solvent extraction method, the extraction temperature, extraction time, solvent composition and the ratio of solvent to material are the main factors, which would affect the extraction rate and yield of resveratrol, and there may be interactions between different factors ${ }^{[35-36]}$, so these factors should be considered comprehensively. Romero-Pérez et al. ${ }^{[37]}$ studied the influence of solvent type, extraction time and extraction temperature on the extraction of resveratrol from grape berry skins. Ethanol/water $(80: 20 \mathrm{v} / \mathrm{v})$, ethanol $100 \%$, ethyl acetate/methanol $(50: 50 \mathrm{v} / \mathrm{v})$, acetone/water $(75: 25 \mathrm{v} / \mathrm{v})$, and acetone $100 \%$ were tested, and the ethanol/water $(80: 20 \mathrm{v} / \mathrm{v})$ was optimized to obtain the highest yield of resveratrol. In addition, the maximum recovery $(>96 \%)$ and satisfactory reproducibility $(6.83-15.13 \%)$ were obtained under the optimal conditions of ethanol/water $(80: 20 \mathrm{v} / \mathrm{v}), 60^{\circ} \mathrm{C}$ and $30 \mathrm{~min}$.

\subsection{Ultrasonic- and microwave-assisted extraction method}

Currently, ultrasonic- and microwave-assisted extraction are regarded as the novel green techniques, which has many advantages over the traditional solvent extraction, such as simple, rapid and time-saving ${ }^{[38-39]}$. In these two methods, power, frequency, extraction time, temperature, solvent combination and the ratio of material to solvent, etc. are the major factors affecting the extraction rate and yield of resveratrol ${ }^{[40-41]}$. Babazadeh et al. ${ }^{[40]}$ have extracted the trans-resveratrol from red grape wastes by the ultrasonic-assisted extraction method. The amounts of trans-resveratrol varied between $68.36 \pm 3.06$ and 862 $\pm 4.67 \mu \mathrm{g} / \mathrm{g}$-dry raw-material of the initial samples based on optimized conditions of $19.4 \mathrm{~min}, 53.6^{\circ} \mathrm{C}$, and ethanol/PEG/water (48:32:20 v/v). Piñeiro et al. ${ }^{[41]}$ have extracted stilbenes (contained trans-resveratrol) from woody material (grape stem and grape cane samples) by the microwave-assisted extraction. A high repeatability and reproducibility (both $>90 \%$ ) were showed under the optimal conditions of $80 \%$ ethanol, $125^{\circ} \mathrm{C}, 750 \mathrm{~W}, 5 \mathrm{~min}$ and sample-solvent ratios between 1:100 and 1:125. In addition, they reported that the microwave-assisted extraction required only around $10 \%$ of the total time when compared to previous methods such as ultrasonicassisted extraction method, etc. It is consistent with the previous report by Zhang et al. ${ }^{[42]}$ that the main advantage of microwave-assisted extraction over ultrasonic-assisted extraction is that the extraction time is shortened. 


\subsection{Supercritical carbon dioxide extraction method}

Supercritical carbon dioxide extraction has become one of the most popular green extraction techniques nowadays. Supercritical carbon dioxide has excellent properties, such as low critical point, non-toxicity, high diffusivity, and low cost ${ }^{[43-44]}$. The fluid pressure, temperature and the co-solvent were essential parameters in supercritical carbon dioxide extraction ${ }^{[45]}$. Casas et al. ${ }^{[45]}$ have extracted the resveratrol from the pomace of Palomino fino grapes by supercritical carbon dioxide extraction. The optimum recovery of resveratrol from the raw material was obtained under the conditions of 400 bar, $35^{\circ} \mathrm{C}$ and $5 \%$ Ethanol, and this recovery was higher than conventional extraction with a solvent mixture at atmospheric pressure [Methanol: $\mathrm{HCl}(0.1 \%)]$. In addition, the main problem encountered in the use of supercritical carbon dioxide is that the less effective in extracting polar compounds from plant materials by its low polarity. However, some reports have already demonstrated that the addition of a small amount of cosolvent (e.g., methanol, ethanol, water) that modifies the polarity of the solvent system allows the extraction of such polar substances ${ }^{[44]}$.

\subsection{Enzymatic hydrolysis method}

Enzymatic hydrolysis method is different from the general resveratrol extraction methods. It is a green method with higher selectivity and efficiency than the chemical synthesis and microbial transformation ${ }^{[46-47]}$.

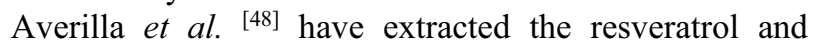
antioxidants from the grape peel by the enzymatic hydrolysis method. The optimum overall extractability of resveratrol from the grape peel was obtained under the conditions of pre-heating grape peel at $95^{\circ} \mathrm{C}$ for 10 $\min , \quad$ a mixture of exo-1,3- $\beta$-glucanase and pectinases at $50^{\circ} \mathrm{C}$ for $60 \mathrm{~min}$. This method has provided a potential low-cost green processing for the industrial fortification of food products with resveratrol. By contrast, some literature reports illustrated that although enzymatic hydrolysis method increased the concentration of resveratrol, subsequent separations still used conventional solvent extraction methods and it needed to be concentrated before further purification, which consumed a large number of organic solvents and time. So a variety of new extraction techniques, such as ultrasound-assisted extraction and microwave-assisted extraction, have been used to extract the resveratrol after the enzymatic hydrolysis method ${ }^{[49]}$.

\section{Functional activities of resveratrol}

Resveratrol exhibits a lot of functional activities such as antioxidant activity, antimicrobial activity, antitumor activity, and prevention of cardiovascular disease.

\subsection{Antioxidant activity}

Resveratrol is a natural polyphenols compound with 3 phenolic hydroxyl groups, so it has strong antioxidant activity. Resveratrol is able to scavenge free radicals produced by human metabolism and protect human organs from undergoing oxidative damage through free radical mediated reactions ${ }^{[50]}$. Fukui et al. ${ }^{[51]}$ founded that resveratrol's neuroprotective effect was dependent on its ability to selectively induce the expression of mitochondrial superoxide dismutase to reduce mitochondrial oxidative stress and damage. In addition, resveratrol can cross the blood brain barrier and exerts potent antioxidant features. Researches have suggested that resveratrol also exerts neuroprotective properties by up-regulating several detoxifying enzymes related to iron proteins ${ }^{[52]}$. Meanwhile, resveratrol can prevent the accumulation of chronic oxidative stress when stress causes hormone secretion disorders. It protects insulin sensitivity of skeletal muscle by improving activities of mitochondrial complexes and antioxidant defense status [53]. Moreover, resveratrol can elicit neuroprotective effects through free radical scavenging and protect human from motor coordination impairment, hydroxyl radical overloading, and neuronal loss [54]. A large number of relevant studies have confirmed that resveratrol can reduces the level of reactive oxygen species and free radicals produced by stress, thereby protecting the organism from oxidative damage.

\subsection{Antimicrobial activity}

Studies have shown that resveratrol has an anti-Listeria monocytogenes effect, and its minimum inhibitory concentration against Listeria monocytogenes was 200 $\mu \mathrm{g} / \mathrm{mL}^{[55]}$. In addition, resveratrol also exhibits a potent anti-bacterial biofilm activity. The minimum inhibitory concentration of resveratrol against Escherichia coli was $456 \mu \mathrm{g} / \mathrm{mL}{ }^{[56]}$. The anti-Escherichia coli activity of resveratrol is achieved by inhibiting bacterial protein expression. When the concentration of resveratrol reaches $200 \mu \mathrm{g} / \mathrm{mL}$, it can interfere and induce bacterial DNA fragmentation. Resveratrol at $25-50 \mu \mathrm{g} / \mathrm{mL}$ inhibited the growth of 5 kinds of dermatophytes such as Trichophyton mentagrophytes, Trichophyton tonsurans, Trichophytonrubrum, Epidermophyton floccosum, and Microsporum gypseum [57]. Resveratrol of low concentration $(20 \mu \mathrm{g} / \mathrm{mL})$ could penetrate into the cell without fatal cell membrane damage but induce apoptosis in Candida albicans ${ }^{[58]}$. Therefore, resveratrol has great potential in the development of antibacterial products.

\subsection{Antitumor activity}

Resveratrol is a natural polyphenols and antitumor agent. It can treat and prevent cancer through a variety of molecular mechanisms such as inducing apoptosis, regulating cell-cycle, inhibiting cytochrome enzyme and cyclooxygenase, and inhibiting the production of cytokine. 
Resveratrol is a novel selective and strong inhibitor of cytochrome enzymes to regulate human cytochrome P450 1B1 (CYP1B1) and kill cancer cells ${ }^{[59]}$. CYP1B1 is known to be involved in the metabolic activation of many diverse environmental procarcinogens, including polycyclic aromatic hydrocarbon, heterocyclic and aryl amines, and nitroaromatic hydrocarbons. Moreover, CYP1B1 expression has been observed at a higher level in various hormone-mediated cancers such as breast, ovarian, endometrial, and prostate ${ }^{[60]}$.

Nawaz et al. ${ }^{[61]}$ founded that in lung cancer cell lines, methylated resveratrol derivative induced cancer apoptosis by caspase- 9 and caspase- 3 activation and blocking poly (ADP-ribose) polymerase, reducing the potential of the mitochondrial membrane and causing cancer cell dysfunction. Research by Jehan et al. [62] showed that resveratrol induced apoptosis by inhibiting cell viability. Angiogenesis usually exist in the pathological and physiological process, and it plays an important role in the proliferation and migration of tumor cells. Inhibiting angiogenesis will prevent the growth and migration of cancer cells. Resveratrol can reduce the level of vascular endothelial growth factor (VEGF) in ovarian cancer cells to inhibit angiogenesis, and then inhibit the growth and proliferation of cancer cells ${ }^{[63]}$.

The antitumor mechanism of resveratrol still needs to be studied in depth, and its application in food, health products and medicines will be further developed in order to provide more methods for humans to prevent and treat cancer.

\subsection{Prevention of cardiovascular disease}

Resveratrol has a certain preventive effect on cardiovascular diseases. At present, there are more and more studies on the cardiovascular protection of resveratrol. The protective effects of resveratrol on the cardiovascular disease mainly include a reduction in platelet aggregation, dilatation of blood vessels, antiatherosclerotic effects, lowering of lipid peroxidation, reduction in endothelin-1, protection of endothelial cells against apoptosis and improvement of serum cholesterol profile and triglyceride concentrations ${ }^{[64]}$. Hung et al. ${ }^{[65]}$ used a rat model of ischemia/reperfusion and found that the incidence and duration of ventricular fibrillation and ventricular tachycardia were significantly reduced in rat myocardium after resveratrol pretreatment. Fukuda et al. [66] founded that the expression of tyrosine kinase receptor, vascular endothelial growth factor receptor, and vascular endothelial cell growth factor in a rat model of myocardial infarction after resveratrol pretreatment was significantly increased.

At present, the research of resveratrol on cardiovascular protection is limited to laboratory research, and clinical research data is relatively rare. There is no uniform standard for safe and effective dosage, and further research is needed. In-depth research on resveratrol will provide strong support for drug development and clinical application for the prevention and treatment of cardiovascular disease.

\section{Nanocarriers of resveratrol}

Resveratrol, as a natural polyphenol, has physiological functions such as anti-oxidation, antibacterial, antitumor, etc. It has attracted wide attention from scientists. However, resveratrol has a poor water solubility, easy to degrade under light, poor oral digestion and absorption, and low bioavailability. Therefore, it is necessary to choose a suitable nanocarrier to embed and protect it to improve its bioavailability.

\subsection{Resveratrol-loaded nanoparticles}

Nanotechnology is a comprehensive technical system that uses nanoscale materials for cross-research and industrialization. Nanoparticle is a kind of colloidal dispersion systems with particle sizes between 1-1000 $\mathrm{nm}$. Drugs exist in the interior and surface of nanoparticles in the form of encapsulation, adsorption and dispersion. Due to the small size and unique structure of nanoparticles, they can increase the dispersibility and digestion residence time of poorly soluble drug molecules in the gastrointestinal tract. Therefore, it is an effective way to solve the low oral bioavailability of poorly soluble drugs.

The current materials for preparing resveratrol nanoparticles are generally degradable materials, mainly including polyorthoesters, polycaprolactone, polyalkyl cyano-acrylate, polylactic acid, polyglycolicacid, polydium clactic-co-glycolic acid and so on. Jung et al. [67] prepared resveratrol-loaded polyethylene glycolpolylactic acid (PEG-PLA) polymer nanoparticles to improve the physicochemical stability and controlled delivery, and investigated its anti-cancer effects on CT26 colon cancer cells in vitro and in vivo experiments. They found that resveratrol nanoparticles showed a stronger ability to inhibit tumor cell proliferation than free resveratrol. Sanna et al. ${ }^{[68]}$ prepared resveratrol-loaded nanoparticles by a nanoprecipitation method. The results of in vitro release test performed that nano-resveratrol had good sustained-release characteristics. Cytotoxicity experiments showed that resveratrol nanoparticles significantly improved the cytotoxicity toward all three prostate cancer cell lines. In addition, natural biodegradable materials are also used as carriers to prepare nanoparticles, mainly proteins carrier materials, including bovine serum albumin and human serum albumin. Guo et al. [69] successfully fabricated resveratrol-bovine serum albumin nanoparticles, which can induce apoptosis of human ovarian cancer SKOV3 cells.

\subsection{Resveratrol-loaded nanoemulsion}

Generally speaking, emulsions contain an oil phase and an aqueous phase. One phase is dispersed in the other phase in the form of droplets. The dispersed phase is called the continuous phase or the external phase, and the dispersed phase is called the dispersed phase or the internal phase. The size of the emulsion particle is related to its concentration, composition, and the type 
and concentration of emulsifier. The emulsification principle of nanoemulsion is basically similar to that of traditional emulsion. The difference is that the particle size of nanoemulsion is smaller, generally ranging from 10 to $100 \mathrm{~nm}$. Sessa et al. ${ }^{[70]}$ used sugar ester P1670, defatted soy lecithin Solec FS-B and polysorbate Tween 20 as the emulsifiers and peanut oil as the oil phase to prepare resveratrol emulsion. By comparing the above emulsions with different particle sizes, they found that ordinary resveratrol emulsions do not easily pass through cell membranes, while resveratrol nanoemulsion is more easily absorbed by the human body through cell membranes. Therefore, using an encapsulated resveratrol into nanoemulsion-based delivery system can improve the bioavailability of resveratrol. Hemar et al. ${ }^{[71]}$ used polyglycerol polyricinoleate as a surfactant, canola oil as the oil phase, and whey protein as an emulsifier to construct a water-in-oil-in-water multiple emulsion to encapsulate resveratrol. The particle size of the emulsion remained basically unchanged with storage at $23{ }^{\circ} \mathrm{C}$ for up to 2 weeks. It indicated that the system has higher stability and greater potential in food applications. Davidov-Pardo et al. ${ }^{[72]}$ used a mixed oil of grape seed oil and orange oil with resveratrol as the oil phase, and Tween 80 as a surfactant. The resulting organic phase was added to an aqueous phase over a $10 \mathrm{~min}$ period under constant magnetic stirring at ambient temperature. By the ultraviolet light (UV-light) test, it is found that encapsulation of resveratrol improved its chemical stability after exposure to UV-light significantly, $88 \%$ retention in nanoemulsions compared to $50 \%$ in dimethylsulphoxide. This study showed that nanoemulsion-based delivery systems have a good protective effect on resveratrol and can reduce its degradation rate after exposed to UV-light.

\subsection{Resveratrol-loaded solid lipid nanoparticles}

Solid lipid nanoparticles is a kind of solid colloidal particle delivery systems prepared by using natural or synthetic solid lipids such as triacylglycerol, lecithin, etc. as a carrier, with a particle size ranging from 10-1000 $\mathrm{nm}$, and embedding or encapsulating drugs into the lipid core. Evren et al. ${ }^{[73]}$ confirmed that resveratrol-loaded solid lipid nanoparticles have a high degree of biocompatibility with normal human skin fiber cells when the concentration of resveratrol is $50 \mu \mathrm{M}$ in the cell experiments. And at this concentration, it can effectively reduce the accumulation of active oxygen and exert antioxidant activity. Teskac et al. ${ }^{[74]}$ fabricated solid lipid nanoparticles by melt-emulsification method to load resveratrol, and measured the cell absorption, transport and internalization of resveratrol. The results showed that resveratrol embedded in solid lipid nanoparticles can improve the cell absorbability. However, the long-term storage stability of solid lipid nanoparticles is poor, and the loading capacity of drug is low $(1 \% \sim 5 \%)$.

\subsection{Resveratrol-loaded nanosuspension}

Nanosuspension is a kind of nano-colloid dispersion systems formed by using emulsifiers to stabilize drug particles. Its advantages are increasing the dispersion of the drug in the aqueous phase and the suspension stability of the drug, and improving the bioavailability of the drug to improve medicinal efficacy. Kobierski et al. [75] prepared resveratrol nanosuspension by high-pressure homogenization technology, using Tween 80, Poloxamer 188, Plantacare 2000 and Inutec SP1 as stabilizers, and its particle size range is $150-220 \mathrm{~nm}$. It can effectively improve the solubility and dissolution rate of resveratrol.

\section{Conclusion}

Resveratrol is a polyphenol that is hardly soluble in water and has important physiological activities. It can be extracted from natural plants by solvent extraction, microwave- and ultrasonic-assisted extraction, supercritical carbon dioxide extraction, and enzymatic hydrolysis method. Some scientific experiments have shown that resveratrol has antioxidant, antibacterial, antiumor, and cardiovascular disease prevention effects. However, due to poor water solubility, easy degradation and low oral bioavailability, resveratrol needs to be embedded inside nano-carriers such as nanoparticles, nanoemulsions, solid lipid particles, and nanosuspensions. With the advancement of science and technology, the research on resveratrol has gradually deepened, and more effective methods of preparing resveratrol can be developed in the future, such as using microbial fermentation method to produce resveratrol, using genetic engineering and cell engineering to transform plants to increase the content of resveratrol, or producing resveratrol through chemical synthesis and transformation methods. On the other hand, nanodelivery carriers with better performance can be developed to encapsulate and protect resveratrol, such as multi-material composite nanoparticles, core-shell structured nanoparticles, and microemulsions and microgels with better stability.

\section{References}

1. D. Delmas, A. Lançon, D. Colin, B. Jannin, N. Latruffe, Curr. Drug Targets. Resveratrol as a chemopreventive agent: a promising molecule for fighting cancer, 7(4), 423-442 (2006)

2. L. Bavaresco, Drugs Exp. Clin. Res. Role of viticultural factors on stilbene concentrations of grapes and wine, 29(5-6), 181 (2003)

3. B. B. Aggarwal, A. Bhardwaj, R. S. Aggarwal, N. P. Seeram, S. Shishodia, Y. Takada, Anticancer Res. Role of Resveratrol in Prevention and Therapy of Cancer: Preclinical and Clinical Studies, 24(5A), 2783-2840 (2004)

4. C. H. Cottart, V. Nivet-Antoine, C. LaguillierMorizot, J. L. Beaudeux, Mol. Nutr. Food Res. 
Resveratrol bioavailability and toxicity in humans, 54(1), 7-16 (2010)

5. J. Constant, Clin. Cardiol. Alcohol, ischemic heart disease, and the French paradox, 20(5), 420-424 (1997)

6. Y. Wang, F. Catana, Y. Yang, R. Roderick, R. B. van Breemen, J. Agric. Food Chem. An LC-MS method for analyzing total resveratrol in grape juice, cranberry juice, and in wine, 50(3), 431-435 (2002)

7. J. Burns, T. Yokota, H. Ashihara, M. E. Lean, A. Crozier, J. Agric. Food Chem. Plant foods and herbal sources of resveratrol, 50(11), 3337-3340 (2002)

8. M. Athar, J. H. Back, X. Tang, K. H. Kim, L. Kopelovich, D. R. Bickers, A. L. Kim, Toxicol. Appl. Pharmacol. Resveratrol: a review of preclinical studies for human cancer prevention, 224(3), 274-283 (2007)

9. M. S. Baliga, S. Meleth, S. K. Katiyar, Clin. Cancer Res. Growth inhibitory and antimetastatic effect of green tea polyphenols on metastasis-specific mouse mammary carcinoma $4 T 1$ cells in vitro and in vivo systems, 11(5), 1918-1927 (2005)

10. A. Amri, J. C. Chaumeil, S. Sfar, C. Charrueau, J. Control. Release. Administration of resveratrol: what formulation solutions to bioavailability limitations, 158(2), 182-193 (2012)

11. M.J. O'Neil, P.E. Heckelman, C.B. Koch, K.J. Roman, C.M. Kenny, M.R. D'Arecca, The Merck Index (fourteenth ed. Merck Publishing, Whitehouse Station, 2006)

12. E. Scott, W. P. Steward, A. J. Gescher, K. Brown, Mol. Nutr. Food Res. Resveratrol in human cancer chemoprevention-choosing the 'right'dose, 56(1), 713 (2012)

13. B. C. Trela, A. L. Waterhouse, J. Agric. Food Chem. Resveratrol: isomeric molar absorptivities and stability, 44(5), 1253-1257 (1996)

14. Y. Schneider, F. Vincent, B. Duranton, et al. Cancer Lett. Anti-proliferative effect of resveratrol, a natural component of grapes and wine, on human colonic cancer cells, 158(1), 85-91 (2000)

15. E. H. Siemann, L. L. Creasy, Am. J. Enol. Vitic. Concentration of the phytoalexin resveratrol in wine, 43(1), 49-52 (1992)

16. A. Csiszar, Ann.NY Acad.Sci. Anti-inflammatory effects of resveratrol: possible role in prevention of age-related cardiovascular disease, 1215, 117(2011)

17. G. Lanzilli, A. Cottarelli, G. Nicotera, S. Guida, G. Ravagnan, M. P. Fuggetta, Inflammation. Antiinflammatory effect of resveratrol and polydatin by in vitro IL-17 modulation Inflammation, 35(1), 240248 (2012)

18. X. Z. Li, X. Wei, C. J. Zhang, X. L. Jin, J. J. Tang, G. J. Fan, B. Zhou, Food Chem. Hypohalous acidmediated halogenation of resveratrol and its role in antioxidant and antimicrobial activities, 135(3), 1239-1244 (2012)
19. E. Fibach, E. Prus, N. Bianchi, et al. Int. J. Mol. Med. Resveratrol: Antioxidant activity and induction of fetal hemoglobin in erythroid cells from normal donors and $\beta$-thalassemia patients, 29(6), 974-982 (2012)

20. D. Bonnefont-Rousselot, Nutrients. Resveratrol and cardiovascular diseases, 8(5), 250 (2016)

21. A. Y. Berman, R. A. Motechin, M. Y. Wiesenfeld, M. K. Holz, npj Precis. Oncol. The therapeutic potential of resveratrol: a review of clinical trials, 1(1), 1-9 (2017)

22. Y. R. Li, S. Li, C. C. Lin, Biofactors. Effect of resveratrol and pterostilbene on aging and longevity, 44(1), 69-82 (2018)

23. V. C. Thipe, K. P. Amiri, P. Bloebaum, et al. Int. J. Nanomed. Development of resveratrol-conjugated gold nanoparticles: interrelationship of increased resveratrol corona on anti-tumor efficacy against breast, pancreatic and prostate cancers, 14, 4413 (2019)

24. A. Acevedo-Fani, H. D. Silva, R. Soliva-Fortuny, O. Martín-Belloso, A. A. Vicente, Food Hydrocolloids. Formation, stability and antioxidant activity of foodgrade multilayer emulsions containing resveratrol, 71, 207-215 (2017)

25. T. Cardoso, A. Gonçalves, B. N. Estevinho, F. Rocha, Powder Technol. Potential food application of resveratrol microparticles: Characterization and controlled release studies, 355, 593-601 (2019)

26. G. F. Balata, E. A. Essa, H. A. Shamardl, S. H. Zaidan, M. A. Abourehab, Drug Des. Dev. Ther. Self-emulsifying drug delivery systems as a tool to improve solubility and bioavailability of resveratrol, 10, 117 (2016)

27. T. Walle, Ann.NY Acad.Sci. Bioavailability of resveratrol, 1215(1), 9-15 (2011)

28. A. Francioso, P. Mastromarino, A. Masci, M. d'Erme, L. Mosca, Med. Chem. Chemistry, stability and bioavailability of resveratrol, 10(3), 237-245 (2014)

29. S. Yang, H. Gao, Pharmacol. Res. Nanoparticles for modulating tumor microenvironment to improve drug delivery and tumor therapy, 126, 97-108 (2017)

30. C. Hong, Y. Dang, G. Lin, et al. Int. J. Pharm. Effects of stabilizing agents on the development of myricetin nanosuspension and its characterization: an in vitro and in vivo evaluation, 477(1-2), 251-260 (2014)

31. R. B. Greenwald, Y. H. Choe, J. McGuire, C. D. Conover, Adv. Drug Deliv. Rev. Effective drug delivery by PEGylated drug conjugates, 55(2), 217250 (2003)

32. W. Li, S. Feng, Y. Guo, Nanomedicine. Tailoring polymeric micelles to optimize delivery to solid tumors, 7(8), 1235-1252 (2012)

33. W. Li, J. Li, J. Gao, et al. Biomaterials. The finetuning of thermosensitive and degradable polymer 
micelles for enhancing intracellular uptake and drug release in tumors, 32(15), 3832-3844 (2011)

34. A. C. Santos, I. Pereira, M. Pereira-Silva, et al. Trends Food Sci. Technol. Nanocarriers for resveratrol delivery: Impact on stability and solubility concerns, 91, 483-497 (2019)

35. J. E. Cacace, G. Mazza, J. Agric. Food Chem. Extraction of anthocyanins and other phenolics from black currants with sulfured water, 50(21), 59395946 (2002)

36. C. Liyana-Pathirana, F. Shahidi, Food Chem. Optimization of extraction of phenolic compounds from wheat using response surface methodology, 93(1), 47-56 (2005)

37. A. I. Romero-Pérez, R. M. Lamuela-Raventós, C. Andrés-Lacueva, M. C. de la Torre-Boronat, J. Agric. Food Chem. Method for the quantitative extraction of resveratrol and piceid isomers in grape berry skins. Effect of powdery mildew on the stilbene content, 49(1), 210-215 (2001)

38. M. C. Guamán-Balcázar, W. Setyaningsih, M. Palma, C. G. Barroso, Appl. Acoust. Ultrasoundassisted extraction of resveratrol from functional foods: cookies and jams, 103, 207-213 (2016)

39. S. Pimentel-Moral, I. Borrás-Linares, J. LozanoSánchez, D. Arráez-Román, A. Martínez-Férez, A. Segura-Carretero, J. Pharm. Biomed. Anal. Microwave-assisted extraction for Hibiscus sabdariffa bioactive compounds, 156, 313-322(2018)

40. A. Babazadeh, A. Taghvimi, H. Hamishehkar, M. Tabibiazar, Food Biosci. Development of new ultrasonic-solvent assisted method for determination of trans-resveratrol from red grapes: Optimization, characterization, and antioxidant activity (ORAC assay), 20, 36-42 (2017)

41. Z. Piñeiro, A. Marrufo-Curtido, C. Vela, M. Palma, Food Bioprod. Process. Microwave-assisted extraction of stilbenes from woody vine material, 103, 18-26 (2017)

42. H. F. Zhang, X. H. Yang, Y. Wang, Trends Food Sci. Technol. Microwave assisted extraction of secondary metabolites from plants: Current status and future directions, 22(12), 672-688 (2011)

43. S. Al Jitan, S. A. Alkhoori, L. F. Yousef, Studies in Natural Products Chemistry. Phenolic acids from plants: extraction and application to human health, 58, 389-417 (Elsevier , 2018)

44. C. Mantell, M. Rodríguez, E. Martinez de la Ossa, Eng. Life Sci. A screening analysis of the high-pressure extraction of anthocyanins from red grape pomace with carbon dioxide and cosolvent, 3(1), 38-42 (2003)

45. L. Casas, C. Mantell, M. Rodríguez, et al. J. Food Eng. Extraction of resveratrol from the pomace of Palomino fino grapes by supercritical carbon dioxide, 96(2), 304-308 (2010)

46. L. H. Quan, J. W. Min, Y. Jin, C. Wang, Y. J. Kim, D. C. Yang, J. Agric. Food Chem. Enzymatic biotransformation of ginsenoside Rb1 to compound $K$ by recombinant $\beta$-glucosidase from Microbacterium esteraromaticum, 60(14), 37763781 (2012)

47. Z. Wang, L. C. Zhao, W. Li, L. X. Zhang, J. Zhang, J. Liang, Molecules. Highly efficient biotransformation of polydatin to resveratrol by snailase hydrolysis using response surface methodology optimization, 18(8), 9717-9726 (2013)

48. J. N. Averilla, J. Oh, Z. Wu, et al. J. Sci. Food Agric. Improved extraction of resveratrol and antioxidants from grape peel using heat and enzymatic treatments, 99(8), 4043-4053 (2019)

49. L. Zhou, B. Jiang, T. Zhang, S. Li, Food Biosci. Ultrasound-assisted aqueous two-phase extraction of resveratrol from the enzymatic hydrolysates of Polygonum cuspidatum, 31, 100442 (2019)

50. M. Leopoldini, T. Marino, N. Russo, M.Toscano, J. Phys. Chem. A. Antioxidant properties of phenolic compounds: H-atom versus electron transfer mechanism, 108(22), 4916-4922 (2004)

51. M. Fukui, H. J. Choi, B. T. Zhu, Free Radic. Biol. Med. Mechanism for the protective effect of resveratrol against oxidative stress-induced neuronal death, 49(5), 800-813 (2010)

52. M. Mokni, S. Elkahoui, F. Limam, M. Amri, E. Aouani, Neurochem. Res. Effect of resveratrol on antioxidant enzyme activities in the brain of healthy rat, 32(6), 981-987 (2007)

53. J. Zheng, L. L. Chen, H. H. Zhang, X. Hu, W. Kong, D. Hu, Metabolism. Resveratrol improves insulin resistance of catch-up growth by increasing mitochondrial complexes and antioxidant function in skeletal muscle, 61(7), 954-965 (2012)

54. K. T. Lu, M. C. Ko, B. Y. Chen, et al. J. Agric. Food Chem. Neuroprotective effects of resveratrol on MPTP-induced neuron loss mediated by free radical scavenging, 56(16), 6910-6913 (2008)

55. S. Ferreira, F. Domingues, J. Sci. Food Agric. The antimicrobial action of resveratrol against Listeria monocytogenes in food-based models and its antibiofilm properties, 96(13), 4531-4535 (2016)

56. D. Hwang, Y. H. Lim, Sci Rep. Resveratrol antibacterial activity against Escherichia coli is mediated by Z-ring formation inhibition via suppression of FtsZ expression, 5, 10029 (2015)

57. M. M. Y. Chan, Biochem. Pharmacol. Antimicrobial effect of resveratrol on dermatophytes and bacterial pathogens of the skin, 63(2), 99-104(2002)

58. J. Lee, D. G. Lee, Curr. Microbiol. Novel antifungal mechanism of resveratrol: apoptosis inducer in Candida albicans, 70(3), 383-389 (2015)

59. Y. J. Chun, S. Kim, D. Kim, S. K. Lee, F. P. Guengerich, Cancer Res. A new selective and potent inhibitor of human cytochrome P450 $1 B 1$ and its application to antimutagenesis, 61(22), 8164-8170 (2001) 
60. Y. J. Chun, Y. K. Oh, B. J. Kim, D. Kim, S. S. Kim, H. K. Choi, M. Y. Kim, Toxicol. Lett. Potent inhibition of human cytochrome P450 1B1 by tetramethoxystilbene, 189(1), 84-89 (2009)

61. W.Nawaz, Z. Zhou, S. Deng, X. Ma, X. Ma, C. Li, $\mathrm{X}$. Shu, Nutrients. Therapeutic versatility of resveratrol derivatives, 9(11), 1188 (2017)

62. A. R. Hussain, S. Uddin, R. Bu, O. S. Khan, S. O. Ahmed, M. Ahmed, K. S. Al-Kuraya, PLoS One. Resveratrol suppresses constitutive activation of AKT via generation of ROS and induces apoptosis in diffuse large B cell lymphoma cell lines., 6(9), e24703 (2011)

63. A. B. Tino, K. Chitcholtan, P. H. Sykes, A. Garrill, J. Ovarian Res. Resveratrol and acetyl-resveratrol modulate activity of VEGF and $I L-8$ in ovarian cancer cell aggregates via attenuation of the $N F-\kappa B$ protein, 9(1), 1-12 (2016)

64. M. A. Markus, B. J. Morris, Clin. Interv. Aging. Resveratrol in prevention and treatment of common clinical conditions of aging, 3(2), 331 (2008)

65. L. M. Hung, J. K. Chen, S. S. Huang, R. S. Lee, M. J. Su, Cardiovasc. Res. Cardioprotective effect of resveratrol, a natural antioxidant derived from grapes, 47(3), 549-555 (2000)

66. S. Fukuda, S. Kaga, L. Zhan, D. Bagchi, D. K. Das, A. Bertelli, N. Maulik, Cell Biochem. Biophys. Resveratrol ameliorates myocardial damage by inducing vascular endothelial growth factorangiogenesis and tyrosine kinase receptor Flk-1, 44(1), 43-49 (2006)

67. K. H. Jung, J. H. Lee, J. W. Park, C. H. T. Quach, S. H. Moon, Y. S. Cho, K. H. Lee, Int. J. Pharm. Resveratrol-loaded polymeric nanoparticles suppress glucose metabolism and tumor growth in vitro and in vivo, 478(1), 251-257 (2015)

68. V. Sanna, I. A. Siddiqui, M. Sechi, H. Mukhtar, Mol. Pharm. Resveratrol-Loaded Nanoparticles Based on Poly (epsilon-caprolactone) and Poly (d, l-lactic-co-glycolic acid)-Poly (ethylene glycol) Blend for Prostate Cancer Treatment, 10(10), 38713881 (2013)

69. L. Guo, Y. Peng, Y. Li, et al. Oncol. Lett. Cell death pathway induced by resveratrol-bovine serum albumin nanoparticles in a human ovarian cell line, 9(3), 1359-1363 (2015)

70. M. Sessa, M. L. Balestrieri, G. Ferrari, et al. Food Chem. Bioavailability of encapsulated resveratrol into nanoemulsion-based delivery systems, 147, 42$50(2014)$

71. Y. Hemar, L. J. Cheng, C. M. Oliver, L. Sanguansri, M. Augustin, Food Biophys. Encapsulation of resveratrol using water-in-oil-in-water double emulsions, 5(2), 120-127 (2010)

72. G. Davidov-Pardo, D. J. McClements, Food Chem. Nutraceutical delivery systems: resveratrol encapsulation in grape seed oil nanoemulsions formed by spontaneous emulsification, 167, 205-212 (2015)

73. E. H. Gokce, E. Korkmaz, E. Dellera, G. Sandri, M. C. Bonferoni, O. Ozer, Int. J. Nanomed. Resveratrol-loaded solid lipid nanoparticles versus nanostructured lipid carriers: evaluation of antioxidant potential for dermal applications, 7, 1841 (2012)

74. K. Teskač, J. Kristl, Int. J. Pharm. The evidence for solid lipid nanoparticles mediated cell uptake of resveratrol, 390(1), 61-69 (2010)

75. S. Kobierski, K.Ofori-Kwakye, R. H. Müller, C. M. Keck, Pharmazie. Resveratrol nanosuspensions for dermal application-production, characterization, and physical stability, 64(11), 741-747 (2009) 